\title{
Pavlovian conditioning of an approach bias in low-dependent smokers
}

\author{
Roy Thewissen • Remco C. Havermans • \\ Nicole Geschwind • Marcel van den Hout • Anita Jansen
}

Received: 10 November 2006 / Accepted: 30 April 2007 / Published online: 24 May 2007

(C) Springer-Verlag 2007

\begin{abstract}
Rationale In the present study, it was investigated whether smokers can acquire a behavioural approach bias through Pavlovian conditioning.

Objectives More specifically, it was tested whether pairing neutral stimuli with either smoking availability or unavailability would lead to both differential urge responding to these stimuli and a corresponding shift in approach bias. Materials and methods Thirty-nine low-dependent smokers performed a stimulus-response compatibility (SRC) task with which one can determine an approach bias. Next, participants received a conditioning session in which one cue (either a blue or yellow background screen colour) was paired with the opportunity to smoke (CS+) and another cue was paired with the absence of the opportunity to smoke (CS-). After conditioning, all participants again performed the SRC task.

Results Evidence for the conditioning of an approach bias but not smoking urges was found. This effect, although, was only apparent when smokers had been prompted to determine the contingency between the cues and smoking outcome. Conclusions It is concluded that one can differentially condition an approach bias in low-dependent smokers.
\end{abstract}

\footnotetext{
R. Thewissen

Department of Medical, Clinical and Experimental Psychology, Maastricht University,

Maastricht, The Netherlands

R. Thewissen • R. C. Havermans $(\bowtie) \cdot$ N. Geschwind $\cdot$ A. Jansen Department of Experimental Psychology, Faculty of Psychology, Maastricht University,

P.O. Box 616, 6200 MD Maastricht, The Netherlands

e-mail: r.havermans@psychology.unimaas.nl

M. van den Hout

Department of Clinical Psychology, Utrecht University,

Utrecht, The Netherlands
}

Keywords Urge to smoke - Smoking availability Incentive motivation · Differential conditioning . Contingency awareness

Most addiction researchers agree that addictive behaviour is the result of learning processes. Addictive behaviour is learned through experience with addictive substances. One particular form of such learning through experience comprises Pavlovian conditioning, the learning of an association between a conditioned stimulus (CS) and a biologically relevant unconditioned stimulus (US). Pavlovian conditioning has been suggested to play an important role in the development, maintenance and relapse of drug use. According to this view, drug-related stimuli or cues (e.g. a cigarette), become associated with drug intake (e.g. smoke intake), and as a result, these cues will elicit conditioned drug responses or cue reactivity. Cue reactivity comprises subjective responses (e.g. craving), psychophysiological responses (e.g. changes in heart rate and skin conductance level) and behavioural responses (e.g. drug-seeking behaviour; Carter and Tiffany 1999; Drummond 2000; Drummond et al. 2000).

Although it is commonly assumed that cue reactivity is the result of a Pavlovian conditioning history, only a modest number of studies has actually demonstrated this in human subjects (see, e.g. Glautier et al. 1994; Lavez et al. 1999; Hogarth et al. 2003). This appears to be particularly true of behavioural cue reactivity (Glautier and Tiffany 1995). Recently, Mogg et al. (2003) demonstrated that smokers, in comparison with non-smokers, maintained their gaze longer on smoking-related pictures than neutral pictures and that they demonstrated a much stronger behavioural approach bias towards smoke cues in a stimulus-response compatibility (SRC) task (see also Mogg et al. 2005). During this task, participants are instructed to move a manikin figure towards or away from smoking-related and neutral stimuli. 
Responses to stimuli with a positive valence are compatible with a behavioural approach tendency, whereas responses to stimuli with a negative valence are compatible with a behavioural avoidance tendency (see De Houwer 2003). As these measures (attentional bias and behavioural approach bias) correlated, Mogg and colleagues suggest that these response biases are mediated by a common underlying mechanism of incentive motivation as would be predicted by the incentive sensitization model of addictive behaviour (see Robinson and Berridge 1993, 2003). According to this model, drug use leads to neural sensitization of certain brain substrates such as the nucleus accumbens. Through the process of Pavlovian conditioning, cues are associated with the drug, and hence, these cues will acquire incentive salience. Robinson and Berridge termed this process incentive sensitization. According to this interpretation then, the approach bias demonstrated by Mogg et al. (2003) is the result of a Pavlovian learning history. However, such a learning history was not manipulated or controlled in this experiment, which means that the observed approach bias towards smoke cues in smokers can also be explained in terms other than conditioning. For example, the difference in approach bias between smokers and non-smokers may be attributed to individual differences in the familiarity of the smoke cues, or smokers may simply be more sensitive to any arousing stimuli (see Robbins and Ehrman 1992). Robbins and Ehrman (1992) rightfully point out that the claim that cue reactivity results from Pavlovian conditioning requires the demonstration of conditioning of such cue reactivity.

The present study aimed to test whether it is possible to differentially condition an approach bias to initially neutral stimuli in smokers. Further, Hogarth et al. (2006) recently argued that at least some degree of contingency knowledge is required to demonstrate learned motivated behaviour. Reviewing several human nicotine conditioning studies, Hogarth and Duka (2006) convincingly argue that successful conditioning of cue reactivity depends on the development of an explicit drug reward expectancy. Therefore, it was also investigated whether prompting contingency awareness has an effect on differential conditioning of subjective urges to smoke and on the tendency to approach smokingrelated cues.

\section{Materials and methods}

\section{Participants}

Thirty-nine smokers ( 13 men, 26 women; $M$ age $=23.11$ years; $\mathrm{SD}=6.87$ ) who smoked a minimum of five cigarettes a day for at least 2 years were recruited through convenience sampling among the student population of Maastricht
University. The sex ratio of this sample approximates the ratio of male and female students at Maastricht University. All participants completed the Fagerström test for nicotine dependence (FTND; Heatherton et al. 1991). The average score on the FTND was $2.41(\mathrm{SD}=1.89)$, which indicates that participants, on average, had a low level of 'nicotine dependence'. Fifteen participants smoked less than 10 cigarettes a day, 22 participants smoked between 11 and 20 cigarettes a day, and 2 participants smoked between 21 and 30 cigarettes a day. Participants had to abstain from smoking for $2 \mathrm{~h}$ before the experiment. An abstinence period of $2 \mathrm{~h}$ was chosen to avoid floor or ceiling effects of urge to smoke during the conditioning task (see Thewissen et al. 2005).

Independent variables

Smoking cues The smoking cues were stimuli presumed to elicit conditioned urge responses as a result of smoking history. The participant's favourite brand of cigarettes, a lighter and an ashtray were used as smoking cues.

Availability cues A blue or a yellow colour of a computer screen signalled the occurrence or non-occurrence of smoking. For half of the participants, a blue-coloured computer screen indicated that smoking - after presentation of the smoking cues-was allowed, and a yellow-coloured computer screen indicated that smoking was not allowed. For the other half, the meaning of the colours of the computer screens was reversed. These availability cues served as CSs, respectively, predicting the occurrence of smoking (CS+) and absence of smoking (CS-). The US consisted of one puff of a cigarette.

\section{Dependent variables}

Self-reported urge to smoke During the conditioning task, 11-point Likert scales displayed on the computer screen ("At this moment, I feel": 0 "no urge to smoke at all", 10 "an almost irresistible urge to smoke") were used to measure urge to smoke (see Dols et al. 2002 and Thewissen et al. 2005).

SRC task The SRC task the participants had to perform in the present experiment was based on the SRC task described by Mogg et al. (2003) and was programmed in E-prime (Psychology Software Tools). The task comprised two blocks of 80 trials. In each trial, either a $160 \mathrm{~mm}$ high $\times$ $215 \mathrm{~mm}$ wide smoking-related picture (ten different smokingrelated pictures were used, e.g. a picture of a woman smoking a cigarette, a pack of cigarettes, a glass ashtray, a close-up of a hand holding a burning cigarette, et cetera) or neutral picture (ten different neutral pictures were used, e.g. a picture of a 
woman putting on lipstick, a box of crayons, a glass jar, a close-up of a hand holding a pencil, et cetera) was displayed at the centre of the screen together with a manikin (an approximately $18 \mathrm{~mm}$ high $\times 10 \mathrm{~mm}$ wide matchstick figure) placed exactly between the outer border (either the upper or lower border) of the picture and the edge of the screen. For each block, each of these four different trials was presented ten times against a blue background screen color and another ten times against a yellow background, thus rendering a total of eight different trial types.

Each block of trials had a different stimulus response assignment. In one block, the participants were instructed to approach the smoking-related pictures with the manikin by using the up or down arrow keys depending on the starting position of the manikin and to avoid the neutral picture by moving the manikin away from the picture outside the screen. The following trial was initialized upon completion of the correct response (i.e. approach or avoidance), and the time to complete each trial was recorded (in milliseconds). A correct response required a minimum of five consecutive key presses. In the other block, participants received the instruction to avoid the smoking-related pictures and to approach the neutral pictures. The order of these two blocks was counterbalanced between participants per group, and within each block, the eight different trial types were presented in a random order for each separate participant.

Participants performed this SRC task twice. The first task was preceded by a practice session in which the participants were given the opportunity to practice the task at hand. This practice session too comprised two blocks. In one block, they had to approach pictures depicting a chair and to avoid pictures displaying a lamp. This stimulus response assignment was reversed for the other block. Each block comprised 16 trials, and the order of the two blocks was counterbalanced between the participants.

\section{Procedure}

The experiment was approved by a local ethics committee. The experiment started with a brief verbal description of the general procedure of the experiment. After this, all participants also received written information regarding the experiment and were asked to sign a consent form if they still wished to participate. All participants provided informed consent before their participation in the present experiment. In addition, participants completed a general smoking questionnaire and the FTND. Further, end-expired air carbon monoxide (Smokerlyzer, Bedfont Scientific, CO in parts per million [ppm]) was measured before the start of the experiment.

First, participants performed the SRC task, followed by the conditioning task. The conditioning procedure was also programmed in E-prime (Psychology Software Tools) and required participants to follow instructions presented on screen. During the conditioning task, participants were presented with two situations in which they would be exposed to smoking cues, indicated by a blue or yellow background screen colour. Half of the participants received instructions to pay careful attention to stimuli predicting the opportunity to smoke or not (instruction group), whereas the other half did not receive such instructions (no instruction group). Instructions concerning the contingency of the screen colour and smoking opportunity were manipulated, as it has been found that such contingency instructions facilitate conditioning (see, e.g. Hogarth et al. 2006).

The conditioning procedure (adopted from Dols et al. 2002 and Thewissen et al. 2005) thus started with the presentation of a blue or a yellow screen. Participants were instructed to concentrate on the screen for $25 \mathrm{~s}$ and to attend to their urge to smoke, after which they rated their momentary urge to smoke on an 11-point Likert scale presented on the coloured computer screen. Next, a participant's favourite pack of cigarettes, a lighter and an ashtray were placed in front of the computer. The participant was instructed to take one cigarette out of the pack and to handle the cigarette (touch it, place it between their lips, holding it) without lighting the cigarette. After $25 \mathrm{~s}$, the participant again rated his urge to smoke on a second 11-point Likert scale presented on the coloured screen. After the second urge assessment, an instruction would appear on the coloured screen stating that now it was allowed or not allowed to take one puff of a cigarette. This instruction was paired with one of the two screen colours. Per group, half of the participants were allowed to smoke when the background screen colour was yellow and not allowed to smoke when the screen was blue. The other half of the participants received the opposite arrangement.

When the instruction on the computer screen indicated that smoking was allowed, the participant took one puff and exhaled the smoke through a respiratory tube. If the instruction indicated that smoking was not allowed, the participant held the cigarette between his lips and a burning lighter next to it, but did not light the cigarette. In between trials, cigarettes, ashtray and lighter were removed, and participants were instructed to read in a magazine for $2 \mathrm{~min}$ during which the screen colour switched to a neutral grey. Each participant completed a total of eight trials: four trials with a blue-coloured screen and four trials with a yellowcoloured screen presented in a random order with the restriction of no more than three consecutive conditioning trials of the same type. In total, the conditioning task had a duration of approximately $40 \mathrm{~min}$.

Directly after conditioning, participants had to perform the same SRC task as before the conditioning phase. At the end of the experiment, participants were fully debriefed and received a 15-euro voucher for compensation. 


\section{Results}

Data reduction and analyses

The results are reported separately for the two different outcome measures, that is, the self-reported urge to smoke as assessed during the conditioning task and the SRC task. For all analyses, a rejection criterion of 0.05 was used. HuynFeldt epsilon corrections and the corresponding adjustments of the degrees of freedom are reported for all repeatedmeasures analyses in which the assumption of sphericity was violated.

Self-reported urge to smoke within the conditioning phase

Participants repeatedly rated their urge to smoke on 11point Likert scales before and after exposure to the smoking cues while they were exposed to either the availability or the unavailability cue (i.e. the screen colours predicting whether participants would be either allowed or not allowed to smoke after exposure to the smoking cues). Half of the participants had received instructions to pay special attention to predictors of being allowed to smoke; the other half did not receive such instructions. The urge data were analyzed using a 2 (availability cues: availability versus unavailability) $\times 2$ (smoking cues: absent versus present $) \times 4$ (trial: $1,2,3$ or 4 ) $\times 2$ (group: no instructions versus instructions) mixed model analysis of variance (ANOVA). Mean self-reported urge to smoke for the smoking cues (irrespective of the availability cues) as measured during the conditioning task for the 'no instructions' and the 'instructions group' is depicted in Fig. 1.

The analyses confirmed that there was a main effect of smoking cues, $F(1,37)=18.77, p<0.001$. When exposed to the smoking cues, participants reported higher urge scores than in the absence of the smoking cues (see Fig. 1). There was no significant main effect of availability cues, $F(1,37)=$ 0.69 , ns, implying that conditioning of differential urge responding to these cues had not occurred. In other words, participants did not seem to have learned the association between the availability cue (i.e. CS) and smoke intake (i.e. US).

No significant main effect of trial was revealed, $F(1.99$, $70.45)=1.99$, ns. However, there was a significant interaction between smoking cues and trial, $F(3,111)=7.58, p<$ 0.001 , indicating that the effect of the smoking cues changed over the course of the trials. To explore this interaction further, separate post hoc tests were conducted with trial as the independent variable and urge responding in the presence or absence of smoking cues as the dependent variable. Initially, higher urges were reported in the presence of smoking cues compared to their absence, but this difference diminished over trials due to a significant increase in urge to smoke in the absence of smoking cues
$[F(2.03,77.29)=4.67, p<0.02]$, whereas urge responding to the presence of smoking cues remained at the same level over trials $[F(1.97,74.79)<1]$.

SRC task

Due to technical failure, reaction time data from one participant in the no instruction group was not recorded. To remove outliers, reaction times were excluded per participant and per trial type if they were smaller than $200 \mathrm{~ms}$ or larger than $2.5 \mathrm{SD}$ above the mean $(11 \%$ of the data; see also Mogg et al. 2003).

Participants performed the SRC task twice, once before and once after the conditioning procedure. It was expected that participants (all smokers) would be faster to approach smoking-related pictures than to avoid them and that this particular response tendency would be larger than for the neutral pictures. Furthermore, it was expected that at the post-test the magnitude of this effect would be more pronounced in the presence of the availability cue (i.e. the screen color predictive of being allowed to smoke; the $\mathrm{CS}+$ ) than in the presence of the unavailability cue (the CS-).

The data were analyzed using a 2 (test: pre-test versus post-test) $\times 2$ (stimulus: smoke picture versus neutral picture) $\times 2\left(\mathrm{CS}\right.$ : $\mathrm{CS}+$ versus $\left.\mathrm{CS}^{-}\right) \times 2$ (group: no instructions versus instructions) mixed model ANOVA.

The dependent variable was the response tendency calculated per participant and per picture class (smoke and neutral) as the difference in RT between the avoidance response and the approach response. A positive response tendency for smoke pictures would thus reflect relatively more rapid approach than avoidance of smoking-related pictures. Figure 2 displays the mean response tendency towards both the neutral pictures and the smoking-related pictures at pre-test and the post-test for each separate group.

Analyses revealed a main effect of stimulus, $F(1,37)=$ 9.72, $p<0.01$. Replicating results reported by Mogg et al. 2003, 2005 and Bradley et al. (2004), participants had a more positive response tendency towards smoking-related pictures than towards the neutral pictures. Further, we found a significant main effect of group $F(1,37)=4.89, p<$ 0.05 , which is qualified by a significant test $\times \mathrm{CS} \times$ group three-way interaction, $F(1,37)=11.42, p<0.01$. Exploring this interaction, post hoc analyses were conducted separately within each group to test for a potential test $\times \mathrm{CS}$ interaction. No such an interaction was found for group 'no instructions', $F(1,18)=3.50$, ns, but a clear test $\times \mathrm{CS}$ interaction was found in group 'instructions', $F(1,19)=$ $8.15, p<0.01$. This interaction reflects a CS-specific shift in response tendency; that is, at post-test, the participants in the 'instructions' group demonstrated a more positive response tendency towards both the smoking-related and neutral pictures when presented against the CS+ back- 
Fig. 1 Effect of smoking cues (irrespective of availability cues) on self-reported urge to smoke at trials $1-4$ of the conditioning task for the "no instruction group' $(n=19)$ and the 'instruction group' $(n=20)$
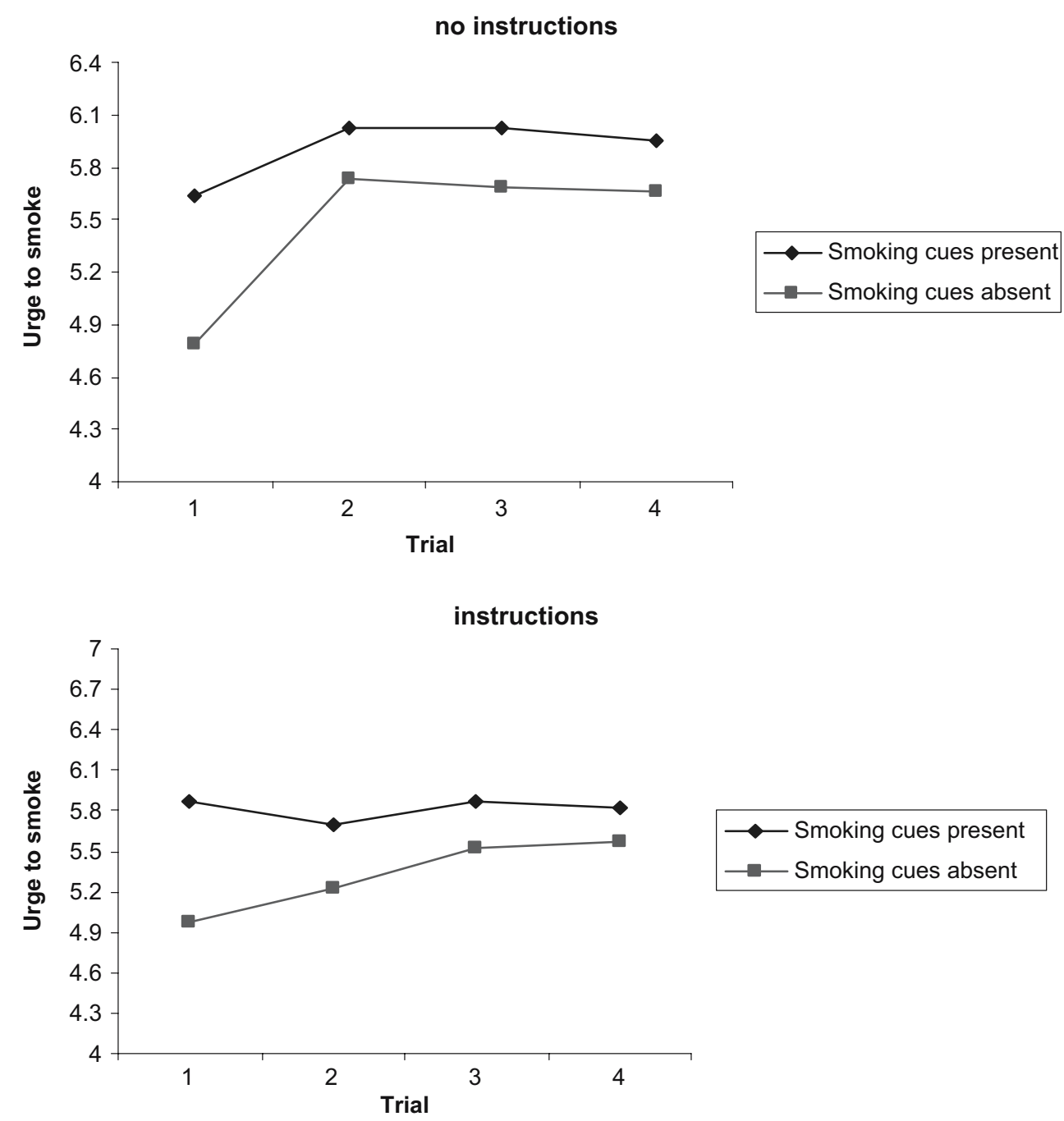

ground as compared to the response tendencies for these pictures when presented against the CS- background. In other words, after the conditioning procedure, the approach tendency was stronger for the $\mathrm{CS}+$ than for $\mathrm{CS}^{-}$, as can also be inferred from Fig. 2. Further post hoc $t$-tests were executed to test for a main effect of CS within the group 'instructions'. It was found that participants from group 'instructions' demonstrated a stronger response tendency towards the $\mathrm{CS}+$ than towards the $\mathrm{CS}-$ after conditioning $[t(19)=2.95, p<0.01]$, whereas no significant difference in response tendency was found between the $\mathrm{CS}+$ and the $\mathrm{CS}-$ before the conditioning trials $[t(19)=-1.34, \mathrm{~ns}]$.

The overall ANOVA also revealed a test $\times$ stimulus $\times$ CS three-way interaction $F(1,37)=4.17, p<0.05$. At pre-test, the response tendency for the neutral pictures was smaller when presented with the CS+ than when presented with the $\mathrm{CS}-$, whereas at post-test, this difference in response tendency towards the neutral pictures was reversed for the CSs. No other effects were found, all $p \mathrm{~s}>0.12$.

\section{Discussion}

The present experiment replicated earlier findings that smokers are faster to approach smoking-related pictures than to avoid them and that this particular approach tendency is larger for smoke pictures than for neutral pictures (Mogg et al. 2003; Bradley et al. 2004; Mogg et al. 2005). Moreover, the present study demonstrates that such an approach bias can be the result of Pavlovian conditioning. One may argue that conditioned approach bias is somewhat of a misnomer, as the CSs in the present study were diffuse background stimuli (i.e. background screen colour). As such, participants could not effectively approach or avoid these stimuli. Further research is required to determine to what degree a conditioned approach bias as demonstrated in the present experiment is location specific. Nonetheless, it is clear that the $\mathrm{CS}+$ at least acquired an excitatory property capable of reliably motivating a response towards the more discrete pictures presented at the centre of the screen. In this 

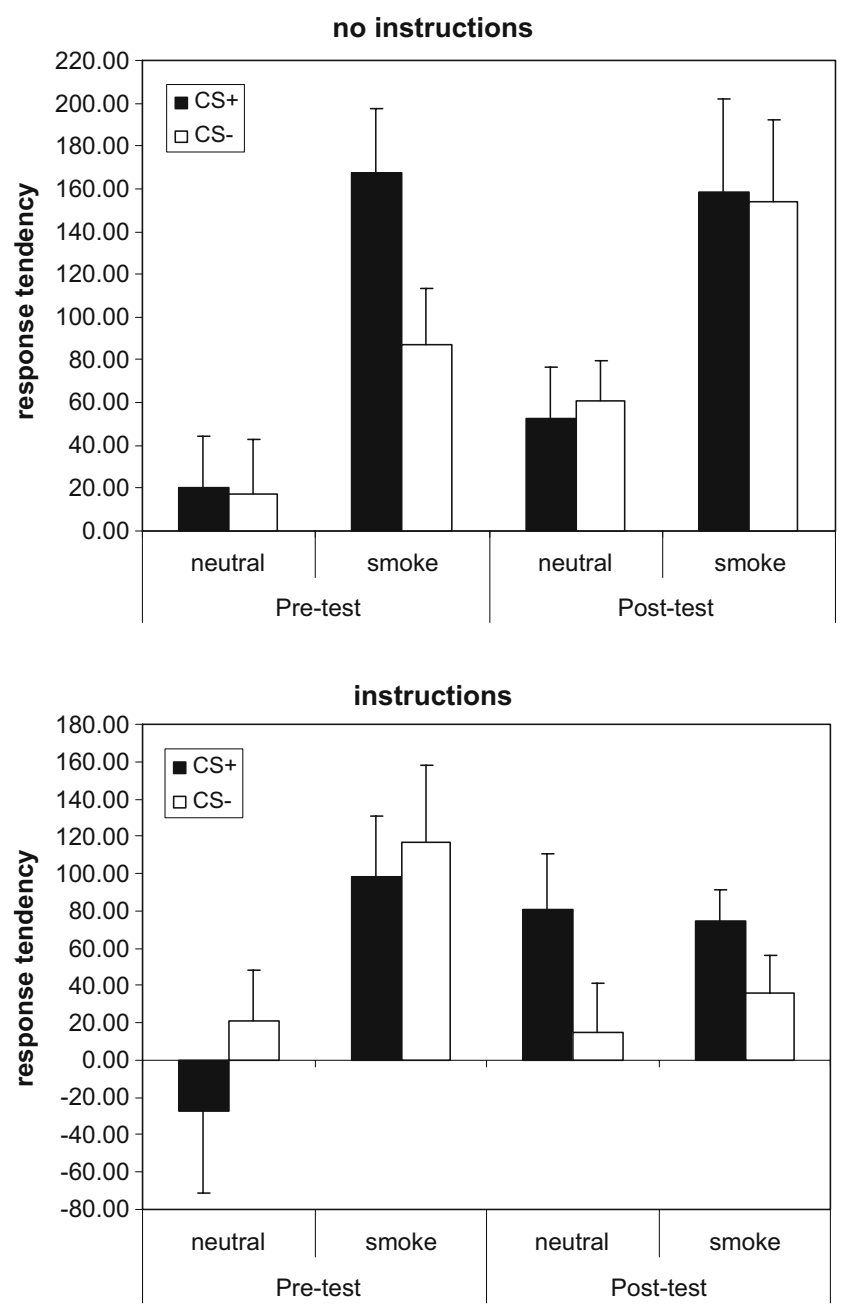

Fig. 2 Mean response tendency (avoid RT minus approach RT) plus standard errors of the mean towards both the neutral pictures and the smoking-related pictures at pre-test and the post-test for the 'no instruction group' $(n=19)$ and the 'instruction group' $(n=20)$

respect, one can argue that the $\mathrm{CS}+$ did come to elicit an approach bias. Interestingly, the content of these pictures being approached appears not to matter after conditioning. This implies that the CS+ acquired the ability to generate approach behaviour irrespective of the presentation of other smoking cues. Conversely, the fact that the approach bias towards the smoke cues when presented with the CS- is smaller at post-test indicates that the CS- acquired the ability to inhibit such approach responding.

This study thus demonstrated differentially conditioned behavioural responding in low-dependent smokers as revealed by a stronger approach tendency when the availability cue $(\mathrm{CS}+)$ was presented than when the unavailability cue (CS-) was presented. This effect, though, was only apparent in the group who received instructions to actively determine the specific availability cues - smoking contingencies. This effect of instructions is in line with the notion that contingency awareness is necessary for learned appeti- tive motivation in humans (see Hogarth et al. 2006). In accordance with expectancy learning theory, Hogarth and Duka (2006) have argued that cue reactivity depends on the development of an explicit drug expectancy and the present pattern of results that conditioning of an approach bias only develops in participants that have had prior contingency prompting corroborates this line of reasoning. These results then also have implications for understanding the SRC task. De Houwer (2003) argues that the SRC task implicitly tests the valence of certain stimuli through the demonstration of a response bias. However, if demonstrating such a response bias hinges on the explicit awareness of the valence of the stimuli presented, one may argue that the bias in itself is explicit and that the SRC task is implicit only in the sense that it indirectly assesses stimulus valence. However, as we did not measure contingency awareness in the present sample of participants, this suggestion remains somewhat speculative and warrants further research.

The results also indicated another difference between the groups 'instructions' and 'no instructions'; that is, the participants that were prompted to pay attention to specific contingencies between the availability cues and smoking demonstrated weaker approach tendencies to the pictures. One may argue that this is the result from the instructions providing a cognitive load during the SRC task. However, the observed difference in approach tendencies between the two groups was not limited to the SRC task at the post-test. The difference in approach tendency was also observed before the conditioning phase. Therefore, this difference is more likely the result of pre-experimental group differences than the manipulation of prompting contingency awareness at the start of the conditioning phase.

Although the data suggests that prompting participants to be contingency aware is necessary to promote conditioning of an approach bias, it was clearly not sufficient to demonstrate differential urge responding to the availability cues. This may reflect the notion that cue-elicited drug responding does not require the experience of subjective craving, as has been suggested by several researchers (see, e.g. Tiffany 1990). However, according to Hogarth and Duka (2006), subjective cue-elicited craving is an index of drug expectancy. Therefore, within group 'instructions', one would have expected some degree of urge to smoke to be differentially controlled by $\mathrm{CS}+$ and $\mathrm{CS}-$ as we found in several other studies using a similar conditioning design (see Dols et al. 2002; Thewissen et al. 2005; Thewissen et al. 2006). We did, however, not explicitly test for participants' contingency awareness, and thus, it is not unlikely that a substantial portion of the participants were not contingency aware (see also Hogarth and Duka 2006). A more straightforward explanation for the present pattern of results, though, is that the subjective cue-reactivity measure in the present study was less sensitive than the approach bias. 
Although we found in previous studies that explicit instructions regarding the contingency between availability cues and smoking does lead to differential urge responding (see, e.g. Thewissen et al. 2005), we typically assessed urge responding with continuous line scales (i.e. visual analogue scales) allowing for a much more fine-grained assessment of momentary urges than on the 11-point Likert scales used in the present study.

Taken together, the results of the present study demonstrate that an approach bias can be differentially conditioned in low-dependent smokers. That is, low-dependent smokers showed a greater approach tendency when presented with a cue signalling the availability of smoking $(\mathrm{CS}+)$, while this approach bias was inhibited when presented with a cue signaling the unavailability of smoking (CS-). This effect, though, was only evident in a group of smokers that was prompted about the contingency between the availability cues and smoking outcome, implying that the approach bias reflects explicit expectancy learning.

Acknowledgement This Research was supported by Grant 985-10006 from NWO-ZON program addiction.

Roy Thewissen, Department of Medical, Clinical and Experimental Psychology, and Department of Experimental Psychology, Maastricht University, The Netherlands. Nicole Geschwind, Remco C. Havermans and Anita Jansen, Department of Experimental Psychology, Maastricht University, The Netherlands. Marcel van den Hout, Department of Clinical Psychology, Utrecht University, The Netherlands. The authors would like to thank Peter de Jong for providing a rough version of the SRC task used in the present study and anonymous reviewers for many helpful suggestions and comments on an earlier version of this article.

\section{References}

Bradley BP, Field M, Mogg K, De Houwer J (2004) Attentional and evaluative biases for smoking cues in nicotine dependence: component processes of biases in visual orientating. Behav Pharmacol 15:29-36

Carter BL, Tiffany ST (1999) Meta-analysis of cue reactivity in addiction research. Addiction 94:327-340

De Houwer J (2003) A structural analysis of indirect measures of attitudes. In: Musch J, Klauer KC (eds) The Psychology of evaluation: affective processes in cognition and emotion. Lawrence Erlbaum, Mahwah, NJ, pp 219-244
Dols M, van den Hout M, Kindt M, Willems B (2002) The urge to smoke depends on the expectation of smoking. Addiction 97:87-93

Drummond DC (2000) What does cue-reactivity have to offer clinical research. Addiction 95(Suppl 2):S129-S144

Drummond DC, Litten RZ, Lowman C, Hunt WA (2000) Craving research: future directions. Addiction 95(Suppl 2):S247-S255

Glautier S, Tiffany ST (1995) Methodological issues in cue reactivity research. In: Drummond DC, Tiffany ST, Glautier S, Remington $\mathrm{B}$ (eds) Addictive behaviour: cue exposure theory and practice. Wiley, Oxford, England, pp 75-97

Glautier S, Drummond DC, Remington B (1994) Alcohol as an unconditioned stimulus in human classical conditioning. Psychopharmacology 116:360-368

Heatherton TF, Kozlowski LT, Frecker RC, Fagerström K (1991) The Fagerström test for nicotine dependence: a revision of the Fagerström tolerance questionnaire. Br J Addict 86:1119-1127

Hogarth L, Duka T (2006) Human nicotine conditioning requires explicit contingency knowledge: is addictive behaviour cognitively mediated? Psychopharmacology 184:553-566

Hogarth L, Dickinson A, Duka T (2003) Discriminative stimuli that control instrumental tobacco-seeking by human smokers also command selective attention. Psychopharmacology 168:435-445

Hogarth L, Dickinson A, Hutton SB, Bamborough H, Duka T (2006) Contingency knowledge is necessary for learned motivated behaviour in humans: relevance for addictive behaviour. Addiction 101:1153-1166

Lavez AB, Herzog TA, Brandon TH (1999) Classical conditioning of environmental cues to cigarette smoking. Exp Clin Psychopharmacol 7:56-63

Mogg K, Bradley BP, Field M, De Houwer J (2003) Eye movement to smoking-related pictures in smokers: relationship between attentional biases and implicit and explicit measures of stimulus valence. Addiction 98:825-836

Mogg K, Field M, Bradley BP (2005) Attentional and approach biases for smoking cues in smokers: an investigation of competing theoretical views of addiction. Psychopharmacology 180:333-341

Robbins SJ, Ehrman RN (1992) Designing studies of drug conditioning in humans. Psychopharmacology 106:143-153

Robinson TE, Berridge KC (1993) The neural basis of drug craving: an incentive sensitization theory of addiction. Brain Res Brain Res Rev 18:247-291

Robinson TE, Berridge KC (2003) Addiction. Annu Rev Psychol 54:25-53

Thewissen R, Van den Hout M, Havermans RC, Jansen A (2005) Context-dependency of cue-elicited urge to smoke. Addiction 100:387-396

Thewissen R, Snijders SJBD, Havermans RC, van den Hout M, Jansen A (2006) Renewal of cue-elicited urge to smoke: implications for cue exposure treatment. Behav Res Ther 44:1441-1449

Tiffany ST (1990) A cognitive model of drug urges and drug-use behavior: Role of automatic and nonautomatic processes. Psychol Rev 97:147-168 